1 Supporting Indigenous Youth Activity Programmes: A Community-Based Participatory

\title{
2 Research Approach
}

3 Angela M. Coppola, * Nicholas L. Holt, \& Tara-Leigh F. McHugh

4 Faculty of Kinesiology, Sport and Recreation, University of Alberta, Edmonton, Alberta, Canada

5 Author Notes

6 *Angela M. Coppola was a doctoral student in the Faculty of Kinesiology, Sport and Recreation

7 at the University of Alberta when the research programme was designed and implemented. She

8 is currently an Assistant Professor of Health Sciences at Indiana University Kokomo.

9 Corresponding Author: Angela M. Coppola, angela.coppola1@gmail.com, coppolaa@iuk.edu,

10 Division of Allied Health Sciences, Indiana University Kokomo, 2300 S. Washington Street,

11 Kokomo, IN, USA.

12 Funding Acknowledgements: The authors would like to acknowledge the Interdisciplinary

13 Health Research Academy at the University of Alberta for providing seed funding for the

14 community consultations in this research programme. The authors would also like to

15 acknowledge the Alberta Centre for Child, Family, and Community Research (now PolicyWise

16 for Children \& Families) for funding the final research and application phases of this programme

17 through the first author's Scobey Hartley Doctoral Award.

18 Partner Acknowledgements: The first author would like to thank the second and third authors for

19 their long-term mentorship that contributed to the quality and impact of this research programme.

20 The authors would like to thank our research programme partners, with a special thank you to

21 Susan Sinclair, who contributed their knowledge and time to this programme. Your prolonged

22 engagement was invaluable. This paper is dedicated to you. 
23 This paper was written in memory of our community partner, Allison Pratley Laird, who was

24 devoted to supporting play and recreation opportunities for all youth. Her insight was integral to

25 the relevancy and impact of this research programme.

26 Notes on contributors

27 Angela M. Coppola, $\mathrm{PhD}$ is an assistant professor in the Division of Allied Health Sciences at

28 Indiana University Kokomo. Her programme of research involves the use of community-based

29 participatory research methods to support health and activity initiatives with diverse youth and

30 communities. She also researches positive body image programmes with and for women.

31 Nicholas L. Holt, $\mathrm{PhD}$ is a professor in the Faculty of Kinesiology, Sport and Recreation at the

32 University of Alberta. He studies psychosocial aspects of participation in sport and physical

33 activity among children, adolescents, and their families.

34 Tara-Leigh F. McHugh, PhD is an associate professor in the Faculty of Kinesiology, Sport and

35 Recreation at the University of Alberta. Her programme of research is focused on better

36 understanding and enhancing the sport experiences of youth. She has extensive experience

37 engaging in community-based participatory research with Indigenous youth. 


\section{Supporting Indigenous Youth Activity Programmes: A Community-Based Participatory}

\section{Research Approach}

40 The purpose of this three-year, multi-phase community-based participatory research (CBPR)

41 programme was to explore how to support Indigenous youth activity programmes and

42 programme planners in Alberta, Canada. This CBPR programme was comprised of five phases:

43 (1) Identifying mutual interests and learning how to partner, (2) Building culturally-relevant

44 activity programmes with and for youth, (3) Defining CBPR programme goals and

45 understanding our roles, (4) Exploring how to support Indigenous youth activity programmes,

46 and (5) Applying and informing practices for supporting Indigenous youth activity programmes.

47 Phases Four and Five of this CBPR are the focus of this paper. Phase Four was an exploration of

48 programme planners' experiences of, and recommendations for, building partnerships and

49 programmes to identify how to support Indigenous youth activity programmes and programme

50 planners. Fifteen programme planners from four urban areas in Alberta participated in one-on-

51 one interviews. Findings are represented by three themes: (1) Building capacity for collaboration

52 and programme planning, (2) Connecting partners and existing programmes, and (3) Aligning

53 and integrating goals and resources with existing programmes. Phase Five, the application phase

54 of this CBPR, involved the co-development of a gathering event to apply areas for support (i.e.,

55 themes) from Phase Four of this CBPR, as well as to reflect on the strengths and challenges of

56 applying such supports. Partners can use implications from the research programme processes

57 and outcomes to explore their role in and practices for supporting these programmes.

58 Keywords: Indigenous youth, Aboriginal youth, activity, holistic health, capacity-building,

59 networking, partnerships, community-based participatory research, qualitative research, action

60 research 
Introduction

62 Activity programmes, which include physical activity (PA), sport, or recreation initiatives, have

63 been described as beneficial and relevant to the holistic development of Indigenous ${ }^{1}$ youth ${ }^{2}$ (e.g.,

64 Hanna 2009; McHugh, Deal, Blye, Dimler, Halpenny, Sivak and Holt 2018). Specifically, youth

65 have described the perceived emotional, physical, mental, and spiritual benefits of activity

66 programmes $^{3}$ (e.g., McHugh et al. 2018). For example, Indigenous youth have shared that

67 mental benefits, such as communication skills, are fostered through sport and recreation

68 (McHugh 2011). Indigenous youth have commonly described their activity experiences as being

69 fun and making them happy, exemplifying the emotional benefits from these experiences (e.g.,

70 Halas, McRae and Petherick 2012). There is also evidence of the important role of cultural

71 activities in this context, such as powwow (Kerpan and Humbert 2015), praying, and teachings

72 from Elders (Sasakamoose, Scerbe, Wenaus and Scandrett 2016), which youth have perceived as

73 spiritual health benefits. Lastly, youth have shared their perceived physical benefits from

74 activity, such as a reduced likelihood for diabetes (Tang, Community Wellness Programmes and

75 Jardine 2016), and the importance of making good health choices to perform an activity well

76 (Petrucka, Bickford, Bassendowski, Goodwill, Wajunta and Yuzicappi et al. 2016).

In addition to the potential holistic health benefits that can be experienced through

78 activity programmes, negative experiences of racism, bullying, or exclusion have also been

79 reported (Bruner, Hillier, Baillie, Lavallee, Bruner and Hare et al. 2016), making the context in

\footnotetext{
${ }^{1}$ The term 'Indigenous' is used when referring to peoples and youth who are native to a land or country. In a Canadian context, this also is inclusive of First Nations, Metís, and Inuit Peoples. ${ }^{2}$ In this paper and research projects cited in this paper, the age of youth reported being involved in activity, sport, and/or recreation programmes and studies ranged from age 5 to 19 years.

3 'Activity programmes' include any planned activities or initiatives that include the promotion of physical activity for youth within a community.
} 
80 which activity occurs important to both youth and programme development. Activity

81 opportunities have the potential to foster spaces for the positive development of Indigenous

82 youth when these spaces promote holistic health, traditional culture and values, connections to

83 the land (e.g., hunting and fishing), and relationships to Indigenous communities (e.g., family

84 and community member support; Bruner et al. 2016; Hanna 2009; McHugh et al. 2018).

85 Furthermore, those promoting or facilitating activity opportunities should also address social

86 (e.g., exclusion, racism), financial (e.g., registration and travel), and physical (e.g., facilities, lack

87 of cultural relevance) barriers to participation (McHugh et al. 2018).

88 Community-based researchers and practitioners have created and/or informed the

89 development of Indigenous youth activity programmes using community-based and collaborative

90 approaches (e.g., Blodgett, Schinke, Fisher, George, Peltier and Ritchie et al., 2008; McHugh,

91 Coppola and Sinclair 2013; Schinke, Yungblut, Blodgett, Eys, Peltier and Ritchie et al. 2010).

92 Collaboration involves relationships between Indigenous youth, communities, and partners ${ }^{4}$ who

93 can put the pieces together to facilitate programme development that elicits positive experiences

94 for Indigenous youth (e.g., Blodgett et al. 2008). Collaboration is particularly important when

95 working with Indigenous communities because of their right to self-determination of decisions

96 and programmes concerning their lives and wellbeing (Smith 2012). For example, McHugh and

97 colleagues (2013) worked with community members to develop relevant PA-based research

98 questions with communities, and findings from this research subsequently informed local

99 Indigenous youth activity programmes. In these collaborative approaches, community members

100 partnered with community-based researchers and/or practitioners whose roles support

\footnotetext{
4 The term "partners" refers to any person or organization who seeks to or is currently collaborating with Indigenous youth activity programmes or programme planners. If a specific partner is being referred to, the type of partner or partnership is elaborated on.
} 
101 community members to inform or develop activity programmes that Indigenous youth and

102 communities may benefit from.

103 The purpose of this three-year, multi-phase community-based participatory research

104 (CBPR) programme was to explore how to support Indigenous youth activity programmes and

105 programme planners in Alberta, Canada. The research questions that guided this CBPR included:

106 (a) What are Alberta Indigenous youth activity programme planners' experiences of building

107 (i.e., co-creating and engaging in) partnerships and programmes? (b) What are Alberta

108 Indigenous youth activity programme planners' recommendations for building partnerships and

109 programmes? Gaining a better understanding of how to support Indigenous youth activity

110 programmes and programme planners can inform community-based practitioners' and

111 policymakers' collaborative practices.

112 This CBPR was created from my $\left(\mathrm{AMC}^{5}\right)$ long-term experiences of working with, and

113 learning from, an Indigenous Elder and youth in urban school-communities. After a couple years

114 of community engagement and reflections of cultural humility (i.e., my constant reflections of

115 my role, and relational and methodological practices in the CBPR; see Coppola \& McHugh 2016

116 for an example), I realised that I was not meant to deliver programme outcomes in communities,

117 rather my role was to learn how to support my research and programme partners in their journey

118 of facilitating or promoting activity programmes among Indigenous youth. In this paper, the five

119 phases of a CBPR programme are described, with Phases Four and Five being the focus of this

120 paper.

121 Community-Based Participatory Research Method

5 'My' or ' $\mathrm{I}$ ' in this paper refers to the first author. 
122 A CBPR framework (Israel et al. 1998) is conceptualised as a partnership approach that

123 equitably involves academic and non-academic partners in all phases of the research process.

124 Each partner contributes their expertise and knowledge to understanding the community issue 125 and enhancing the wellbeing of a community (Israel et al. 1998). Given this is a collaborative 126 method which involves the inclusion of research practices, Israel and colleagues (2005) have 127 offered several principles, such as building relationships and community partnerships, to guide 128 the development and implementation of CBPR. Specific to CBPR with Indigenous Peoples, 129 Fletcher (2003) describes how developing a self-determined research agenda involves the

130 identification of community needs and the discussion and development of a research 131 relationship.

132 As described in the following sections, various research partners were involved 133 throughout the five phases of this CBPR. The research partners identified with diverse ethnic 134 backgrounds including Indigenous backgrounds, such as Cree, Mi’kmaw, and Metís Peoples. 135 Indigenous Peoples and community members have a right to decision-making in research or 136 programmes that concern their community and wellbeing (Smith 2012), and this CBPR approach

137 facilitated the development of a research agenda and the inclusion of community members who 138 participated as research partners throughout the research process. A CBPR approach also aligns 139 with the first author's research paradigm. I identify as a Caucasian-American woman, and a 140 feminist participatory researcher. Specifically, my feminist perspective facilitates a balancing of 141 power dynamics, which is important as I and my ancestors have not experienced colonisation. 142 Feminist research from a participatory approach stresses the need for inclusion, participation, 143 action, social change, research reflexivity, and placing the experiences and perspectives of 144 participants at the core of the research (Frisby, Reid, Millar and Hoeber 2005; Reid 2004). 
145 Conducting research from a feminist participatory research paradigm has enhanced my process

146 of cultural humility (Tervalon \& Murray-Garcia 1998) and building long-term research

147 partnerships with those who have different experiences (e.g., racial marginalisation) and

148 perspectives (e.g., Indigenous ways of knowing) than my own. Thus, my role is to support

149 Indigenous Peoples' and community members' perspectives and voices as partners in CBPR, and

150 to facilitate their engagement and decision-making in the research process. NLH and TLFM

151 served as advisors and mentors to the first author, guiding her through partnership and

152 methodology decisions.

153 While there is no specific set of guidelines for engaging in CBPR, researchers (e.g.,

154 Israel, Eng, Schulz and Parker 2005) have identified five phases to guide the development of a

155 CBPR programme. The phases are broadly named and described, and can be renamed to be

156 context-specific. The first phase is 'partnership formation and maintenance,' which is described

157 as the fundamental phase that involves assessing and reflecting on each members' capacities,

158 learning each partners' needs and interests, and working together to achieve mutual goals, and

159 was renamed in this study as Phase One: Identifying Mutual Interests and Learning how to

160 Partner. During the second 'community assessment and diagnosis' phase, CBPR partners gain

161 an understanding of the relevant needs of the community based on a mutual area of focus or

162 study. CBPR partners also identify a community assessment or consultation procedure. This

163 phase was renamed Phase Two: Building Culturally-Relevant Activity Programmes with and for

164 Youth. The third phase, 'definition of the issue,' involves the use of relevant data collection

165 methods to formally refine the focus of the research partnership. This phase was titled Phase

166 Three: Defining CBPR Programme Goals and Understanding Our Roles. In the fourth phase,

167 'documentation and evaluation of the partnership process,' the research goals and design are 
168 implemented, CBPR partners identify a balance between knowledge and action, and re-assess the

169 mutual benefits of the partnership. This phase was renamed Phase Four: Exploring How to

170 Support Indigenous Youth Activity Programmes. The 'feedback, interpretation, dissemination,

171 and application of results' phase includes the collaborative development of the application of

172 results, and an exploration of the feedback, strengths, and challenges of the application. This

173 phase was contextualised as Phase Five: Applying and Informing Practices for Supporting

174 Indigenous Youth Activity Programmes.

175 Phase One: Identifying Mutual Interests and Learning How to Partner

176 In the Winter of 2012, I began volunteering in a Junior-High/High School Indigenous

177 Studies class taught by Marie (pseudonym), a Cree Elder. I helped her set up and implement

178 activities in the class, however, it was not until I volunteered for a month that we had our first

179 personal conversation. She asked me if I would like to bead with her and the students. Although I

180 have poor craft skills, I agreed to it. After she gave me materials and showed me how to bead, I

181 started working on my own project. Thirty-minutes later, we laughed together for the first time

182 while looking at the result of my poor technique. Over the next couple months, Marie and I

183 started to get to know one another and our mutual interests. We talked about the importance of

184 connecting youth to Indigenous culture and traditions. Alongside the students, I learned about

185 colonisation and the importance of Indigenous cultural revitalisation. Marie and I learned about

186 our areas of interest and expertise. Her interests included traditional games and sport promotion,

187 and connecting youth to culture. I was broadly interested in building relevant activity

188 programmes with and for youth. My capacities or expertise were my knowledge of research

189 practices, and my strengths in developing and facilitating youth sport activities. Her capacities or

190 expertise were traditional Cree knowledges and practices, including traditional games 
191 instruction. She also knew local and relevant issues in the Indigenous youth community in

192 Edmonton as an educator and Elder. Combining our interests, Marie and I worked closely with

193 youth to learn about Indigenous youths' meanings of sport (McHugh et al. 2013).

194 While collaborating to plan a 'sport sampler' event and a photovoice project, we learned

195 how to work and research together. Marie and I had discussions about relevant research and

196 ethical practices in the community as well as university ethics processes. We learned the

197 importance of communication and specifically what each other should know before events or

198 research activities are implemented. For instance, after a miscommunication about data

199 collection, I learned how important it was to discuss recording purposes and, specifically, when

200 and why recording would occur. Fueling our work was our mutual interests and values of

201 cultural knowledge and traditions, and Cree language education through physical activities for

202 youth. The research partners at this time consisted of myself, Marie, and Indigenous youth.

203 Phase Two: Building Culturally-Relevant Activity Programmes with and for Youth

204 In the second phase of this CBPR Marie and I developed an activity programme with and

205 for Indigenous youth. Marie and I met to discuss the goals and components of the programme.

206 First, our programme goal was to promote cultural revitalisation and culturally-relevant sport and

207 PA opportunities for youth. We created the Cree sport programme, wherein I would identify

208 activities for the grade one and two youth, and consult with them (as our partners) to see if they

209 liked the activity. Marie would then translate components of activities into the Cree language.

210 For instance, we planned a jungle obstacle course. Marie translated verbs like jump, throw, and

211 run in the obstacle course instructions so that students could learn the language while engaging

212 in activity. The youth enjoyed a version of the game Gopher Tag. In Gopher Tag, the youth

213 played a regular game of tag and were considered 'safe' if they were in a 'gopher hole' or hula 
214 hoop. If a youth wanted to switch with another in a 'gopher hole,' she would high-five her and

215 say, 'tanisi miscanaskos' which translates to 'Hello, Gopher.'

216 From our perspectives, the strengths of this programme were the ease of implementation,

217 and the youth's consultation and informal feedback. One key challenge was identifying how to

218 promote this type of programming and how community members could partner to share

219 resources, and enhance relevancy and sustainability. In order to address these "how" questions,

220 Marie and I knew we needed to consult with additional community members. We applied for and

221 received a grant from the Interdisciplinary Health Research Academy at the University of

222 Alberta to support the hosting of community consultations. The implementation and outcomes of

223 the community consultations are described in the following third phase of this CBPR

224 programme.

225 Phase Three: Defining CBPR Programme Goals and Understanding our Roles

226 As is recommended by Israel and colleagues (2005), relevant data collection methods

227 were identified and used to formally refine the focus of the CBPR programme. Community

228 consultations were identified by Marie and a newly formed advisory group of Elders and

229 community members as relevant and respectful practices for gathering knowledge. Marie and I

230 provided peace offerings (i.e., blankets, tobacco, and cloth) to the advisory group members, and

231 these members served to guide the purpose, agenda, and traditional ceremony practices for the

232 gatherings. Four community consultations were attended by approximately 30 community

233 members including Elders, Indigenous youth, parents, social workers, teachers, and members

234 from organisations, such as the City of Edmonton, and Alberta Recreation and Parks Association

235 (see Coppola \& McHugh 2016). Ultimately, Marie and I learned through the consultations that 
236 these community members wanted to engage in Indigenous youth activity programmes, but were

237 not sure how to partner with programmes or what programme supports are needed.

238 Marie, myself, and a group of approximately 15 interested consultation participants co-

239 created a research purpose that would support us in addressing this gap in knowledge. We also

240 discussed that my role would be to lead the subsequent "research-focused" phase (i.e., Phase

241 Four described in the following section), and that I would share the findings with our research

242 partners, research participants, and any community members interested in engaging in

243 Indigenous youth activity programmes. The processes of data generation (i.e., interviews), and

244 sampling of participants who have played a role in creating and implementing Indigenous youth

245 activity programmes were also confirmed.

246 Phase Four: Exploring How to Support Indigenous Youth Activity Programmes

247 The purpose of this fourth phase was to understand how to support programmes and programme

248 planners by exploring Indigenous youth activity programme planners' experiences of, and

249 recommendations for, building partnerships and programmes. Fifteen programme planners (12

250 women and three men) from Alberta, Canada were purposefully selected to participate in this

251 phase of the CBPR programme. Purposive sampling involves the recruitment of individuals who

252 can speak to a specific experience to better understand a phenomenon of interest (Mayan 2009).

253 Thus, individuals who had current or previous experiences of co-creating (i.e., collaborating and

254 engaging with partners to develop and implement) Indigenous youth activity programmes were

255 invited to participate. The participants' experiences with activity programmes ranged from five

256 to 20 years, with programming taking place at friendship centres, schools, or non-profit

257 organisations. These programmes promoted overall health, PA, cultural teachings, and self-

258 identity. Participants identified as First Nations, Métis, and non-indigenous Peoples. Their 
259 experiences ranged from school to community programming on reserves near urban areas or in

260 urban areas of Alberta, with a couple participants drawing on experiences in the Northwest

261 Territories as well. Their experiences were rooted in a collaborative approach, and ensured

262 connections to culture and traditions, as well as their respective communities. Pseudonyms are

263 used to protect the participants' identity.

\section{Data Generation}

265 Upon institutional Research Ethics Board approval, all participants engaged in one-on-one semi-

266 structured interviews at their convenience. One-on-one interviews seek the participants'

267 interpretation of their personal experiences of social phenomena (Dicicco-Bloom and Crabtree

268 2006; Mayan 2009), which in this phase was their experiences of building (i.e., co-creating and

269 engaging in) partnerships and programmes. One participant also engaged in a follow-up

270 interview, and three engaged in follow-up email discussions. A semi-structured interview guide

271 was developed based on suggestions from consultation community members from Phase Three,

272 and piloted with an Indigenous youth activity programme planner. The interview guide included

273 questions about creating partnerships and programmes with partners (e.g., How did you and

274 partners co-create the programme? What were the facilitators and challenges? What are your

275 recommendations for creating partnerships and programmes?), and engaging partners in

276 programming (e.g., Please describe your process of engaging community members and youth in

277 this programme).

278 The average interview duration was 66 minutes and approximately 18 hours of interviews

279 including follow-up interviews were generated. Each participant received \$10 amazon.ca e-gift

280 cards for each hour of participation for contributing their time and knowledge. Interviews were

281 conducted face-to-face in the participants' respective communities or via conference call 
282 services. Interviews were audio-recorded and transcribed by a professional transcription agency,

283 and the first author then reviewed the completed transcriptions for accuracy. A reflexive journal

284 was used to be responsive throughout the research process and to document the progression of

285 data generation (Mayan 2009). Thus, interview reflections were noted to highlight the

286 participants' key interview discussion points and messages.

287 Data Analysis

288 Transcripts were analysed using Morse's (1994) four processes of contextualising and

289 recontextualising data for practical use. The first three processes comprised the contextualising

290 phase. Specifically, the first process involves comprehending in which the researcher learns

291 about the setting from the participants and usually begins with data collection and continues

292 through analysis. The first author read and re-read the transcripts to summarise the general

293 message that the participants' conveyed. Then, she coded the transcripts by analysing pieces of

294 participants' descriptions for information that addressed the research purpose. For instance, one

295 participant mentioned that programmes could benefit from reducing pods or silos of initiatives

296 and resources, so this was coded 'reduce pods.' Synthesising the data is a process of merging

297 participants' experiences to describe patterns in the data. Within this study, the first author

298 reviewed the codes to identify patterns, and reflected on how the patterns address the research

299 purpose. For instance, participants' shared specific training and professional development (PD)

300 supports which was synthesised to 'training and PD.' Theorising, or considering why a

301 researcher is seeing specific patterns, involves a review of other theoretical or empirical sources

302 that align with the findings. In this research, CBPR principles most closely related to the

303 experiences and recommendations of programme planners. For example, building capacity is a

304 key principle of CBPR (Israel et al. 2005), and one of the themes or patterns in this research was 
305 the recommendation to offer PD or capacity-building for programme evaluation. The generated

306 knowledge is then used for recontextualising or synthesising into a form that is applicable to the

307 context and other settings. The resulting themes are named and described to be applicable to

308 Indigenous youth activity programme partnership and programme development practices.

309 Results

310 This fourth phase of this research sought to better understand how to support programmes

311 and programme planners by exploring Indigenous youth activity programme planners'

312 experiences of, and recommendations for, building partnerships and programmes. The findings

313 are represented by three themes: (a) Building capacity for collaboration and programme

314 planning, (b) Connecting partners and existing programmes, and (c) Aligning and integrating

315 goals and resources with existing programmes.

316 Building Capacity for Collaboration and Programme Planning

317 The participants described and recommended the need for PD experiences that help them to build

318 capacity to support their programmes. The two key areas identified for building capacity were

319 collaboration and programme planning. In terms of collaboration participants described how

320 communication skills are critical. In building collaborations, Saoirse explained how programme

321 planners should communicate important aspects of their programmes, and can do so using key

322 messages:

323 [You think about] the key parts of your programme and then your longer key messages

324 that are anywhere from 2 to 30 minutes-what's most important for people to understand

325 about our programme to be able to partner with us? So have a bullet list of maybe three or

326 four points for each potential stakeholder that you think you'll have, so what would I say

327 to youth about this?, what would I say to a school teacher about this?, what would I say to 
a parent?, what would I say to a funder?

Programme planning was also identified as an area for building capacity. For instance,

330 when describing the role of programmes in her community, Harper shared that 'Programming

331 and evaluation is important because we [programme planners] have to reflect on what we're

332 doing.' When talking about her experiences of evaluation and identifying programme outcomes

333 for grants, Saoirse said these typically include physical literacy skills, but there are also hard to 334 measure outcomes that she and her partners would like to consider:

The less measureable outcomes, a lot of those come from the informal things like trust being built and just confidence. Like you see kids that show up, they're so shy, and then Taken together, these results provide examples of capacity-building efforts that partners can

341 address in the context of Indigenous youth activity programmes. Because these are specific, it

342 would be beneficial for partners to ensure the relevancy of certain capacity-building activities

343 before providing these supports. This can be achieved by making connections to build

344 partnerships with programme planners, which was the foundation of the second generated theme.

\section{Connecting Partners and Existing Programmes}

346 The participants described the important role of connections between partners and to existing

347 programmes, specifically, through networking and knowledge-sharing. Networking was

348 described as fostering connections between programme planners and potential partners.

349 Knowledge-sharing was described as sharing programme experiences and examples to inform

350 programme practices. For instance, Tiffany described the importance of connections: 
I've always focused my ideas and initial strategies around what the youth would want so of course that has always meant having to have those relationships in place already or having youth interested in talking to me and giving me direction to start with.

354 These connections were described as informal conversations (e.g., asking questions) to get to 355 know one another, or even as larger gatherings to exchange information. As an example of 356 informal connections, when asked about her experiences of engaging youth and community 357 members in programmes, Superwoman said, 'I just like to connect with people on a personal 358 level and, you know, get to know them. And asking them questions and being just in their life, so 359 that's basically like what I do.'

360 Participants also explained how they would benefit by being connected to existing 361 programmes, and being introduced to various activity programme options through

362 demonstrations. For instance, when Cally discussed networking opportunities, specifically, she 363 said:

364 I think having [a] demonstration with things that are happening, like whether it is a traditional game or having those kind of things shared is part of it too, just to give people an idea of what else is happening or some of these projects, and like little tidbits of what

368 Lauren described how she and her programme partners connect with partners through a monthly 369 gathering, and said, 'we just come together and it's all about communication, it's all about how 370 can we [programme planners and partners] help each other, and really it's about how are we 371 better supporting our students?' Networking and knowledge-sharing between partners could 372 involve discussions related to the next theme of aligning and integrating goals and resources with 373 existing programmes. 


\section{Aligning and Integrating Goals and Resources with Existing Programmes}

The participants recommended the need to reduce silos, combine programmes and

resources, and build upon existing programmes. As stated by Joyce, 'the community

organisations aren't connecting with the schools, and the schools are running their own

[programmes].' After describing the issues with recruiting youth for separate programmes

running at the same time, she shared that programme planners 'have a hard [time], 'cause there's no relationship... so I think we [programme planners] should be working together more, and you

know supporting each other more. There needs to be more communication, more interagency work.' The participants shared the importance of knowing existing programme goals and expectations, and your role in the programmes. Bowden said, 'with the programme, you have to find your role,' and went on to say that in the youth centre he worked, he played a supporting role in already existing programmes. He and his partners,' 'role was to support and add on to things, so if someone asked us to help out with this, we [co-programme planners] would be there. It is totally dependent on what's there, and what's going on already.'

Aligning programmes and resources was also described as an important recommendation because without alignment of priorities, there was added stress of sustaining the programme. The participants shared that there was a lack of alignment between some government or funders' priorities and the community priorities. For example, Briana stated:

[The government is] completely ignoring what the community is saying are issues and just focusing on what they think is an issue, and then just forcing the community through funding to provide programming that they think is important or relevant.

The participants shared that clear funding criteria, funding flexibility, combining programmes to share grant resources, and core (i.e., long-term or multi-year) funding would be beneficial to 
397

398

399

400

401

402

403

404

405

406

407

408

409

410

\section{Discussion}

412 The findings from Phase Four of this CBPR provide insight into how to support programmes and

413 programme planners by building capacity, connecting partners, and aligning and integrating

414 programme goals and resources, all of which are integral to partnerships and programmes with

415 and for Indigenous Peoples (e.g., Ball and Janyst 2008; Fletcher 2003; Loppie-Reading and Wien

416 2009). Broadly, capacity-building can be conceptualized as supporting community potential for

417 responding to health issues (e.g., Chino and DeBruyn 2006). Based on the findings, it is

418 recommended that partners begin exploring capacity-building for programme planning (e.g., 
419 evaluation) and collaboration (e.g., communication) skills that programme planners could use in

420 the development and implementation of programmes.

421 Researchers adopting collaborative and participatory approaches draw upon mutual

422 capacity-building (Ball and Janyst 2008) or co-learning (Israel, Schulz, Parker and Becker 2001)

423 in which community members and their partners both share and receive knowledge to build

424 specific skills needed to develop and implement a programme or service. Thus, this type of 425 capacity-building can be adopted to promote programme sustainability (Hacker, Tendulkar, 426 Rideout, Bhuiya, Trinh-Shevrin and Savage et al. 2012). In an indigenous context, mutual 427 capacity-building is encouraged (Ball and Janyst 2008; Chino and DeBruyn 2006) to ensure that 428 partners understand that their expertise (e.g., programme evaluation) is important, but there are 429 knowledge and skills (e.g., traditional practices) to be learned from Indigenous community 430 programme planners and/or members. Taking this into account, it is recommended that partners 431 also explore what knowledge and skills they will need from community members or programme 432 planners in the partnership to establish co-learning and mutual capacity-building.

433 Partnership-building with community members is key to developing Indigenous youth 434 activity programmes (e.g., Blodgett et al. 2010). Therefore, connecting partners and existing 435 programmes, specifically through networking and knowledge-sharing, support and extend upon 436 the partnership-development literature in this context. Specifically, the study findings indicate 437 that partners can support Indigenous youth activity programmes by facilitating connections 438 between existing programmes and between programme planners and potential partners, and these 439 connections might involve opportunities to network and share knowledge. These connections 440 could also occur through informal discussions or through community gatherings or meetings.

441 Ultimately, those supporting programmes can facilitate partnership-building, a key component to 
442 the development in Indigenous youth activity programmes (e.g., Blodgett et al. 2010), by

443 creating connections (e.g., community gatherings, meetings, and informal group discussions that

444 include networking and knowledge-sharing activities) between existing programmes and

445 between programme planners and potential partners.

446 Aligning and integrating programme goals and resources can support programmes and

447 programme planners. Reading and Wien (2009) shared that a silo approach fails to address the 448 health of Indigenous Peoples because it is inconsistent with their historically collectivist

449 worldview and does not take into account the complexities or multiple determinants (e.g.,

450 education, income) of health. Building upon Reading and Wien's (2009) discussion, partners

451 might consider their complementary roles in existing Indigenous youth activity programmes

452 instead of building new and separate programmes with similar missions. Furthermore, aligning

453 programme goals and resources are important to ensure that partners are supporting the needs

454 and priorities of communities and programme planners (e.g., Israel et al. 2005). The Indigenous

455 youth activity programme planners in this study described funding providers as partners.

456 Multiple participants shared that federal funding agencies focused on economic development

457 programmes, however, community members wanted support for other community- or school-

458 based holistic health programmes, which they perceived would facilitate economic development.

459 Thus, funding partners can ensure that their priorities are consistent with community priorities

460 (e.g., holistic health approach; Reading and Wien 2009) or have less strict funding criteria,

461 recognising that Indigenous Peoples and communities should (and have a right to) determine

462 their own initiatives (Smith 2012). Funding partners can also ensure more long-term as opposed

463 to short-term or pilot funding opportunities are available. 
464 Phase Five: Applying and Informing Practices for Supporting Indigenous Youth Activity

465 Programmes

466 You have given life to a purpose and like a child, it needs to be nurtured, keep feeding...the child

467 will mature. - Anonymous participant from Phase Five

468 As recommended by consultation community members in Phase Three, a preliminary analysis

469 and report of the Phase Four research was shared with a group of consultation community

470 members in Phase Three and research participants from Phase Four to facilitate discussions of

471 how to apply the research findings. Such discussions supported the development of the fifth and

472 final "application phase" of this CBPR programme, which involved a gathering event to support

473 Indigenous youth activity programmes in Alberta. Three formal pre-gathering discussions were

474 held with a group of 12 consultation community members from Phase Three and research

475 participants from Phase Four to inform the development of the gathering, including the

476 programme agenda and potential gathering participants. The main goal of the gathering was to

477 apply recommendations (i.e., themes) from Phase Four of this CBPR, as well as to reflect on the

478 strengths and challenges of applying such recommendations.

479 The gathering included approximately 35 attendees from various provincial and

480 community organisations, university, education, and First Nations communities in Alberta. As

481 requested by the pre-gathering discussion participants, an Indigenous leader shared traditional

482 ceremonies, specifically prayer and smudge, to open and close our gathering and acknowledge

483 community protocols. The gathering was held at a community hall in Edmonton, Alberta.

484 Gathering participants were given a conference bag, food for lunch, and refreshments throughout

485 the day. Previous research (e.g., Blodgett, Schinke, Fisher, Yungblut, Recollet-Saikkonen and

486 Peltier et al. 2010; McHugh et al. 2013) has acknowledged the importance of respecting cultural 
487 protocols, and included a feast and refreshments when conducting sharing circles with

488 Indigenous youth to offer thanks for sharing their knowledge. The pre-gathering discussion

489 participants and I also incorporated activity into the agenda to enhance opportunities for informal

490 relationship-building and to simply be active. For instance, a walk in the river valley of

491 Edmonton was an opportunity to engage in informal conversations about each other and our roles

492 in programmes.

Two key components made up the gathering agenda: professional development (PD)

494 speakers and open space technology discussions. The PD session topics (i.e., Connecting with

495 Youth, Sustaining Programmes, and Promoting Stronger Interagency Support) were based on

496 identified needs of participants that were highlighted in Phase Four and during the pre-gathering

497 discussions. For the "Connecting with Youth" session, a community leader with over 12 years of

498 experience engaging with youth as a fitness instructor, and a community athlete and programme

499 developer shared their experiences and strategies for connecting with youth and the community.

500 The "Sustaining Programmes" session included a community-based researcher and evaluator

501 who shared knowledge and resources for conceptualising programme evaluations in a

502 community setting. In the "Promoting Stronger Interagency Support" session, partners in the

503 Mi'Kmaw Physical Activity Leader (MPAL) programme (i.e., a MPAL and the Regional

504 Physical Activity Consultant) shared their experiences of bridging the gap between the Nova

505 Scotia government and Mi'kmaw communities to support PA opportunities. Open space

506 technology (OST; Owen 1993), a highly engaging process whereby the attendees propose topics

507 of discussion and facilitate those discussions with other attendees, was also used to provide a

508 space for discussion of relevant issues related to Indigenous youth activity programmes. 
Feedback was solicited, and twelve gathering participants filled out the feedback form.

510 Gathering participants who provided feedback were primarily from university, non-profit

511 organisations, and social services and heard about the gathering through word-of-mouth or email

512 listserves. They attended the gathering primarily to network, to find support, and to learn from

513 and engage with others. They shared their thoughts on components they found useful and

514 beneficial, their ability to network, their key take-away points, and their next steps in supporting

515 Indigenous youth activity programmes. Using the feedback, OST discussion outcomes, and my

516 personal reflection, I identified outcomes, strengths, challenges, and recommendations for future

517 application. The reflections were guided by the three areas of support identified in Phase Four.

518 The gathering participants who provided feedback reported that they learned and will

519 apply skills and strategies shared in the professional development (PD) sessions, with many

520 asking for future workshops, and one participant even volunteering to plan future gatherings.

521 However, a barrier to understanding capacity-building in this setting was that I was unsure if the

522 gathering participants applied knowledge and ideas even though they indicated they would.

523 Therefore, scheduling follow-up interviews or programme visits is recommended in order to

524 identify the strengths and limitations of applying knowledge in their context, and ultimately to

525 provide long-term support.

526 A welcomed surprise and perceived strength of the gathering was that capacity was not

527 only enhanced during PD sessions, but also during the OST (Owen 1993) discussions. Several

528 gathering participants who provided feedback mentioned that they learned new ideas in OST

529 sessions, and would apply the ideas in their own context, which was the ultimate goal of the

530 gathering. For instance, one participant shared that she would apply learned community

531 engagement strategies from an OST session, saying she took away 'ideas for community 
532 engagement,' and would 'like to apply some of the ideas with the families I work with.' This

533 indicates that pre-planned PD activities are important, but capacity-building also occurs through

534 participant-driven activities and discussions (e.g., OST).

535 OST discussions fostered a space in which participants' voices were heard and respected.

536 When discussing open space discussions, a gathering participant who provided feedback shared

537 that she 'enjoyed them because it offered an open, safe atmosphere.' OST discussions also

538 facilitated genuine knowledge-sharing and networking between programme planners. One

539 gathering participant who provided feedback summarised the relationship between OST and

540 knowledge-sharing clearly, stating:

Open space sessions gave participants a chance to contribute as well as gain. Knowledge exchange is very valuable in each sector, not only for moving forward for 'next steps,' but also just for affirmation and encouragement.

544 It was encouraging to see from participant feedback that this component of the agenda was

545 deemed valuable. In my role, it was important to learn from the research programme partners and

546 implement their ideas. Supporting findings from Bryson and Anderson (2000), a strength of OST

547 is that the technique promotes a participant-driven agenda. A recommendation is to continue 548 exploring the co-development and implementation of OST to support Indigenous youth activity 549 programmes.

550 There was evidence that gathering participants considered their roles when sharing their 551 next steps in programmes. For instance, one gathering participant stated, 'I know I need to show 552 up and be a role model [for youth], we [programme planners] as leaders and individuals need to 553 be part of the change.' Another shared that the most useful, valuable, and beneficial part of the 554 gathering was 'finding out what is going on in Alberta, [the] challenges, and how I can help.' 
555 Therefore, it is recommended to continue prompting and promoting opportunities for partners

556 and programme planners to consider their roles. Whereas role identification was evident, the

557 gathering could have better facilitated the alignment and integration of goals and resources

558 between programmes and partners. For instance, policymakers were invited, and there was a

559 representative from the Ministry of Health. A couple representatives of the Legislative Assembly

560 of Alberta were invited, and one representative RSVP'd, but did not attend. Thus, co-developing

561 a plan for contacting and/or working with government representatives or decision makers to

562 discuss collaboration or policy change to support programme planners is recommended.

\section{General Discussion}

564 This research offers a practical example of responsive participatory research that led to the

565 identification and direct application of areas for supporting Indigenous youth activity

566 programmes. Importantly, the findings from this research are inclusive of the voices of

567 Indigenous peoples, and the research process and outcomes are timely for two main reasons.

568 First, the findings might be useful to policymakers or community-based researchers and

569 practitioners who collaborate with Indigenous Peoples to support and develop Indigenous sport

570 policy, programmes, and initiatives in Canada. Second, the processes and outcomes of this

571 research participatory research programme provide an example of inclusion of underrepresented

572 populations, and a collaborative process of knowledge generation that can deepen our

573 understandings of phenomena in qualitative research in sport, exercise, and health (McHugh

574 2017).

575 The findings of this research contribute to the PA literature by identifying necessary

576 considerations for supporting Indigenous youth activity programmes. Recent research has

577 synthesised the sport and recreation experiences of Indigenous youth (McHugh et al. 2018) and 
578 systematically reviewed research on the positive development of Indigenous youth through sport,

579 activity, or recreation (Bruner et al. 2016). The aforementioned research outlined important

580 factors that should be considered when developing Indigenous youth activity programmes, such

581 as programmes that promote holistic health, integrate culture and traditions, and optimize on

582 community relationships. This CBPR project adds to these recent reviews by identifying areas

583 for supporting programmes and programme planners who work in these contexts (i.e., by

584 building capacity for collaboration and programme planning, connecting partners and existing

585 programmes, aligning and integrating goals and resources with existing programmes, and

586 exploring the implementation of the aforementioned areas for support).

587 In addition to deepening understandings of how to support Indigenous youth activity

588 programmes, this CBPR project also makes an important methodological contribution to the PA

589 literature. I shared detailed descriptions and reflections on how I (as an academic partner)

590 considered my role complementary to the role of my research programme partners. A

591 recommendation for future researchers is to consider including such detail when describing not

592 only CBPR projects, but other equally relational research projects, such as many qualitative

593 studies. Furthermore, co-learning and capacity-building typically evolves as part of a

594 participatory research programme (Israel et al. 2005), but is not always reflected on and reported.

595 Informing McHugh’s (2017) suggestions for deepening our understandings in qualitative

596 research, exploring and reporting these pieces may contribute to a deeper understanding of the

597 necessity and complexity of collaborative research.

598 National, provincial, and local policymakers and community-based practitioners that are

599 hoping to support Indigenous youth activity programmes may benefit from these findings that

600 can inform their roles in developing practices and policies with community members. One future 
601 direction or recommendation is for researchers, community members, and policymakers to

602 collaboratively explore how and where the findings can be shared. Knowledge-sharing or

603 translation is important to bridge the gap between research and practice (CIHR 2014), and doing

604 so with these findings can inform role identification in various positions when collaborating to

605 develop Aboriginal sport, activity or recreation programmes and policies. That said, these

606 findings may have international relevance as, for example, Sport and Recreation Victoria

607 (Australia) promotes the Indigenous Sport and Recreation Programme with a mission to create

608 sport and recreation opportunities that are inclusive of and accessible to Indigenous communities

609 (Victoria State Government, 2017). Therefore, researchers, community-based practitioners, and

610 policymakers in this context in other countries working collaboratively with programme planners

611 may use the findings to explore how to support Indigenous youth activity programmes.

612 A key strength of this research was the rigor that was established as a result of the

613 prolonged engagement with participants (Schinke, Smith and McGannon 2013). I facilitated a

614 democratic and equitable process over time, promoting prolonged engagement of the research

615 programme partners throughout this CBPR (Schinke et al. 2013). This helped me reflect on my

616 responsiveness as a researcher (Morse et al. 2002) in which I would ask myself if research

617 questions, methods, practices were relevant to the research programme partners and addressed

618 the purpose of the research programme. If unsure, I would consult with the research programme

619 partners. Despite the various contributions of this research, it also has some limitations, which

620 have subsequently highlighted areas for future directions. For example, with the exception of one

621 participant, single one-on-one interviews were conducted with participants in Phase Four of this

622 CBPR project. To enhance the depth of experiences shared, future researchers might consider

623 more iterative processes for data generation. As well, Phase Five of this CBPR project also had 
624 some limitations. Despite the invitation of youth to participate in the gathering, there was very

625 little representation from Indigenous youth. The purpose was to discuss how to better support

626 Indigenous youth activity programmes in Alberta, and it would have been beneficial to have

627 more youth voice and representation. In this programme, the Phases Four and Five participants

628 were those who planned programmes with youth. However, Indigenous youth could have

629 provided insight into their basic and holistic health needs, yielding a much richer experience for

630 gathering participants. Thus, exploring youths' involvement and role in programme support

631 initiatives in the future would be beneficial.

632 In conclusion, this research programme's processes and outcomes yielded several

633 implications for supporting Indigenous youth activity programmes, and advancing

634 understandings of collaboration in research, practice, and policy. This research programme

635 outlines CBPR phases, specifically descriptions and reflections of the processes and outcomes of

636 collaboration and identification of areas of support for Indigenous youth activity programmes.

637 Based on the current CBPR process and outcomes, methodological and practical ideas are

638 offered for elaborating on the process of Indigenous youth activity programme development

639 (e.g., Bruner et al. 2016) and participatory research development (McHugh 2017). 


\section{References}

Ball, J., \& Janyst, P. (2008). Enacting research ethics in partnerships with Indigenous communities in Canada: "Do it in a good way." Journal of Empirical Research on Human Research Ethics, 33-51. doi: 10.1525/jer.2008.3.2.33

Blodgett, A. T., Schinke, R. J., Fisher, L., A., George, C. W., Peltier, D., Ritchie, S., \& Pickard, P. (2008). From practice to praxis: Community-based strategies for Aboriginal youth sport. Journal of Sport and Social Issues, 32, 393-414. doi: 10.1177/0193723508323701

Blodgett, A. T., Schinke, R. J., Fisher, L., Yungblut, H. E., Recollet-Saikkonen, D., Peltier, D., Ritchie, S., \& Pickard, P. (2010). Praxis and community-level sport programming strategies in a Canadian Aboriginal reserve. International Journal of Sport and Exercise Psychology, 8, 262-283. doi: 10.1080/1612197X.2010.9671953

Bruner, M. W., Hillier, S., Baillie, C. P. T., Lavallée, L. F., Bruner, B. G., Hare, K., Lovelace, R., \& Lévesque, L. (2016). Positive youth development in Aboriginal physical activity and sport: A systematic review. Adolescent Research Review, doi: 10.1007/s40894-0150021-9

Bryson, J. M., \& Anderson, S. R. (2000). Applying large-group interaction methods in the planning and implementation of major change efforts. Public Administration Review, 60, 143-162.

Canadian Institutes of Health Research (CIHR). (2014). About knowledge translation and commercialization. Available from: http://www.cihr-irsc.gc.ca/e/29418.html [Accessed 27 January 2014].

Chino, M., \& DeBruyn, L. (2006). Building true capacity: Indigenous models for indigenous communities. American Journal of Public Health, 96, 596-599. doi: 10.2105/AJPH.2004.053801

Coppola, A. M., \& McHugh, T-L. F. (2016). Considering culturally-relevant practices and knowledge-sharing when creating an activity-promoting community research agenda. Sport, Education, and Society, 23, 14-27. doi: 10.1080/13573322.2015.1129942

Dicicco-Bloom, B., \& Crabtree, B. F. (2006). The qualitative research interview. Medical Education, 40, 314-321. doi: 10.1111/j.1365-2929.2006.02418.x

Fletcher, C. (2003). Community-based participatory research relationships with Aboriginal communities in Canada: An overview of context and process. Pimatisiwin: A Journal of Aboriginal and Indigenous Community Health, 1, 27-62.

Frisby, W., Reid, C. J., Millar, S., \& Hoeber, L. (2005). Putting "participatory" into participatory forms of action research. Journal of Sport Management, 19, 367-386.

Hacker, K., Tendulkar, S. A, Rideout, C., Bhuiya, N., Trinh-Shevrin, C., \& Savage, C. P., Grullon, M., Strelnick, H., Leung, C., \& DiGirolamo, A. (2012). Community capacitybuilding and sustainability: Outcomes of community-based participatory research. Progress in Community Health Partnerships: Research, Education, and Action, 6, 349360. Doi: 10.1353/cpr.2012.0048

Halas, J., McRae, H., \& Petherick, L. (2012). Advice for physical education teachers from Aboriginal youth: Become an ally. Physical \& Health Education Journal, 78(3), 6-11.

Hanna, R., (2009). Promoting, developing, and sustaining sports, recreation, and physical activity in British Columbia for Aboriginal youth. Document created for First Nations Health Society. Available from: http://www.fnhc.ca/pdf/Sports_Recreation_and_Physical_Activity_ BC_Aboriginal_Youth.pdf [Āccessed 15 April 2013]. 
Israel, B. A., Eng, E., Schulz, A.J. \& Parker, E. A. (2005). Introduction to methods in community-based participatory research for health. In B. A. Israel, E. Eng, A. J. Schulz, \& E. A. Parker (Eds.), Methods in Community-Based Participatory Research for Health (pp. 3-26). San Francisco, CA: Jossey-Bass.

Israel, B. A., Schulz, A. J., Parker, E. A., \& Becker, A. B. (1998). Review of community-based research: Assessing partnership approaches to improve public health. Annual Review of Public Health, 19, 173-202. doi: 10.1146/annurev.publhealth.19.1.173

Israel, B. A., Schulz, A. J., Parker, E. A., \& Becker, A. B. (2001). Community-based participatory research: Policy recommendations for promoting a partnership approach in health research. Education for Health, 14, 182-197.

Kerpan, S., \& Humbert, L. (2015). Playing together: The physical activity beliefs and behaviors of urban Aboriginal youth. Journal of Physical Activity and Health, 12, 1409-1413. doi:10.1123/jpah.2014-0533

Loppie-Reading, C., \& Wein, F. (2009). Health inequalities and social determinants of Aboriginal Peoples' health. Retrieved (April 8, 2015) from: http://www.nccahccnsa.ca/docs/social\%20determinates/nccah-loppie-wien_report.pdf.

Mayan, M. J. (2009). Essentials of qualitative inquiry. Walnut Creek, CA: Left Coast Press, Inc. National Aboriginal Health Organization, n.d. Publications: Terminology. Available from: http://www.naho.ca/publications/topics/terminology/ [Accessed 10 April 2013].

McHugh, T-L.F. (2011). Physical activity experiences of Aboriginal youth. Native Studies Review, 20, 7-26.

McHugh, T-L. (2017). Thinking about the future: Challenges and possibilities. In B. Smith \& A. C. Sparkes (Eds.), Routledge Handbook of Qualitative Research in Sport and Exercise (pp. 445-449). New York, NY: Routledge.

McHugh, T-L. F., Coppola, A. M., \& Sinclair, S. (2013). An exploration of the meanings of sport to Aboriginal youth: A photovoice approach. Qualitative Research in Sport, Exercise, and Health, 5, 291-311. doi: 10.1080/2159676X.2013.819375

McHugh, T-L. F., Deal, C. J., Blye, C-J., Dimler, A. J., Halpenny, E. A., Sivak, A., \& Holt, N. L. (2018). A meta-study of qualitative research examining sport and recreation experiences of Indigenous youth. Qualitative Health Research, 1-13. doi: $10.1177 / 1049732318759668$

McHugh, T-L., F., Kingsley, B. C., \& Coppola, A. M. (2013). Enhancing the relevance of physical activity research by engaging Aboriginal peoples in the research process. Pimatisiwin: A Journal of Aboriginal and Indigenous Health, 11, 293-305.

Morse, J. M. (1994). "Emerging from the data: The cognitive process of analysis in qualitative inquiry. In J.M. Morse (Ed.), Critical issues in qualitative research methods (pp. 23-43). Thousand Oaks, CA: Sage.

Morse, J., et al., 2002. Verification strategies for establishing reliability and validity in qualitative research. International journal of qualitative methods, 1 (2), 1-19.

Owen, H. (1993). Open space technology: A user's guide. Retrieved from, http://elementaleducation.com/wp-content/uploads/temp/OpenSpaceTechnology-UsersGuide.pdf.

Petrucka, P., Bickford, D., Bassendowski, S., Goodwill, E-W., Wajunta, E-C., Yuzicappi, E-B., Yuzicappi, L., Hackett, P., Jeffery, B., \& Rauliuk, M. (2016). Positive leadership, legacy, lifestyles, attitudes, and activities for Aboriginal youth: A wise practices approach for positive Aboriginal youth futures. International Journal of Indigenous Health, 11, 177197. doi: 10.18357/ijih111201616017 
Reid, C. J. (2004). Advancing women's social justice agendas: A feminist action research framework. International Journal of Qualitative Methods, 3(3). Article 1. Retrieved June 21, 2015 from http://www.ualberta.ca/ iiqm/backissues/3 3/html/reid.html

Robbins, J. A., \& Dewar, J. (2011). Traditional Indigenous approaches to healing and the modern welfare of traditional knowledge, spirituality and lands: A critical reflection on practices and policies taken from the Canadian Indigenous example. The International Indigenous Policy Journal, 2. Retrieved September 27, 2015 from: http://ir.lib.uwo.ca/iipj/vol2/iss4/2 DOI: $10.18584 /$ iipj.2011.2.4.2

Sasakamoose, J., Scerbe, A., Wenaus, I., \& Scandrett, A. (2016). First Nation and Métis youth perspectives of health: An Indigenous qualitative inquiry. Qualitative Inquiry, 22, 636-650. doi:10.1177/1077800416629695

Schinke, R. J., Smith, B., \& McGannon, K. R. (2013). Pathways for community research in sport and physical activity: Criteria for consideration. Qualitative Research in Sport Exercise and Health, 5, 460-468. doi: 10.1080/2159676X.2013.846274

Schinke, R., Yungblut, H., Blodgett, A., Eys, M., Peltier, D., \& Ritchie, S. et al. (2010). The role of families in youth sport programming in a Canadian aboriginal reserve. Journal of Physical Activity and Health, 7, 156-166.

Smith, L. T. (1999; 2012). Decolonizing methodologies: Research and Indigenous peoples. London: Zed Books Ltd.

Sport and Recreation Victoria. (2017). Aboriginal sport and recreation. Retrieved August 10, 2018 from: http://sport.vic.gov.au/our-work/participation/inclusive-sport-andrecreation/aboriginal-sport-and-recreation

Tang, K., Community Wellness Program, \& Jardine, C. G. (2016). Our way of life: Importance of indigenous culture and tradition to physical activity practices. International Journal of Indigenous Health, 11, 211-227. doi: 10.18357/ijih111201616018

Tervalon, M., \& Murray-Garcia, J. (1998). Cultural humility versus cultural competence: A critical distinction in defining physician training outcomes in multicultural education. Journal of Healthcare for the Poor and Underserved, 9, 117-125. doi: 10.1353/hpu.201 0.0233

Thorne, S. (2008). Interpretive description. Walnut Creek, California: Left Coast Press Inc. Thorne, S., Reimer-Kirkham, S., \& O'Flynn-Magee, K. (2004). The analytic challenge in interpretive description. International Journal of Qualitative Methods, 3, 1-12. 